\title{
INFERRING INITIAL SPIN PERIODS FOR NEUTRON STARS IN COMPOSITE REMNANTS
}

\author{
E. VAN DER SWALUW ${ }^{1}$ AND Y. Wu ${ }^{2}$ \\ Received 2001 March 14; accepted 2001 May 25; published 2001 June 22
}

\begin{abstract}
We propose a method to infer the initial spin periods of pulsars residing in composite supernova remnants. Such a remnant consists of both a plerionic and a shell-type component, corresponding to the pulsar wind nebula driven by the spin-down luminosity of the central pulsar and the blast wave bounding the supernova remnant, respectively. Theoretical investigations including hydrodynamical simulations have shown that, at late times $(\sim 1000-10,000 \mathrm{yr})$, a simple scaling law connects the radius of the supernova shell to the radius of the plerion. The energy content of the plerion and the total mechanical energy of the supernova remnant enter into this scaling law. One can use this scaling law to estimate the initial spin periods of pulsars residing in composite remnants. We discuss potential pitfalls of this method, including the effect of a small remnant age and of strong radiative losses in the plerion.
\end{abstract}

Subject headings: hydrodynamics — pulsars: general — supernova remnants

\section{INTRODUCTION}

Is a typical neutron star born spinning close to breakup $\left(P_{0} \sim 0.8 \mathrm{~ms}\right.$; Haensel, Salgado, \& Bonazzola 1995)? The answer to this question reveals much about the angular momentum evolution of neutron star progenitors (Heger, Langer, \& Woosley 2000) as well as about the processes that occur during the supernova explosion (Spruit \& Phinney 1998; Lai \& Goldreich 2000). If the initial spin rate is close to the breakup rate, the nascent neutron star may undergo a Rossby wave instability (Lindblom, Owen, \& Morsink 1998) and emit gravitational radiation. Moreover, a rapidly rotating neutron star can obtain a substantial kick velocity through the electromagnetic rocket effect (Harrison \& Tademaru 1975). This can provide a natural explanation for the apparent alignment between the spin axis and pulsar proper motion (Lai, Chernoff, \& Cordes 2001) in the Crab (Caraveo \& Mignani 1999) and Vela pulsars (Pavlov et al. 2000).

A number of studies have been dedicated to the determination of initial spin rates. Those few young pulsars that have measured braking indexes and estimated ages seem to possess initial spin periods from 19 ms (Crab; Lyne, Pritchard, \& Graham-Smith 1993) to 63 ms (PSR B1509-58; Kaspi et al. 1994). ${ }^{3}$ Since only rapidly rotating pulsars have measurable braking indexes, these values may not be representative of the general population. For these, Phinney \& Blandford (1981) and Vivekanand \& Narayan (1981) have developed the pulsar-current analysis. Unfortunately, this method is subject to small-number statistics (Lorimer et al. 1993). Analysis based on pulsar luminosity functions (Emmering \& Chevalier 1989; Narayan 1987) raised the possibility of "injection": the majority of the pulsars may be born with periods as slow as several hundred milliseconds. This hypothesis is corroborated by the apparent paucity of plerions around neutron stars (Srinivasan, Dwarakanath, \& Bhattacharya 1984). A plerion derives energy from the rotational energy of the central pulsar. The overall conclusion from pulsar studies seems to favor a slow initial spin rate.

In this Letter, we propose a new method for inferring the initial spin rates of some neutron stars-the small population that re-

\footnotetext{
${ }^{1}$ Astronomical Institute, Utrecht University, P.O. Box 80000, 3508 TA Utrecht, Netherlands; e.vanderswaluw@ astro.uu.nl.

${ }^{2}$ Canadian Institute for Theoretical Astrophysics, University of Toronto, 60 St. George Street, Toronto, ON M5S 3H8, Canada; wu@ cita.utoronto.ca.

${ }^{3}$ The LMC X-ray pulsar J0537-6910 (16 ms; Marshall et al. 1998) should be born at shorter than $10 \mathrm{~ms}$ for reasonable values of the braking index.
}

sides within composite supernova remnants (SNRs). A composite SNR includes both a plerionic (filled-centered) component (Weiler \& Panagia 1978) and a shell component. The first corresponds to the pulsar wind nebula $(\mathrm{PWN})$, while the latter corresponds to the blast wave of the SNR propagating through the interstellar medium (ISM). They are observationally distinguished by a different spectral power-law index at radio frequencies. The dynamics of such a composite system has been extensively studied (e.g., Reynolds \& Chevalier 1984; Chevalier \& Fransson 1992).

Recently, van der Swaluw et al. (2001) presented hydrodynamical simulations and analytical arguments that explicitly relate the radius of the PWN to that of the SNR. These authors find that the two radii are roughly proportional some time after the initial explosion, with the proportionality constant determined by the ratio of the pulsar spin-down energy and the total mechanical energy of the supernova event. This relation forms the theoretical basis for the current Letter. In this Letter, we follow van der Swaluw et al. (2001) in restricting ourselves to spherically symmetric systems.

\section{EVOLUTION OF A PWN IN AN SNR}

As a pulsar spins down, its rotational energy is largely deposited into the surrounding medium, driving a relativistic pulsar wind into the tenuous bubble of stellar debris left behind by the supernova blast wave. We approximate the spin-down luminosity of the pulsar wind by that of a rotating magnetic dipole:

$$
L(t)=L_{0} /(1+t / \tau)^{2} .
$$

The total spin-down energy is $E_{\mathrm{sd}}=L_{0} \tau$. We neglect the dynamical influence of the pulsar wind on the SNR, assuming that $E_{\text {sd }}$ is well below the total mechanical energy of the supernova explosion, $E_{0} \sim 10^{51}$ ergs.

The evolution of a PWN inside an SNR can be divided into two stages (Reynolds \& Chevalier 1984; Chevalier \& Fransson 1992). In the first stage, the PWN expands supersonically within the bubble blown out by the SNR. The second stage commences at $t \equiv t_{\mathrm{ST}}$ when the PWN encounters the reverse shock of the SNR. The reverse shock heats the interior of the SNR and causes the expansion of the PWN to become subsonic. In this stage the expansion of the PWN is regulated by the expansion of the SNR, which is described by a self-similar Sedov-Taylor solution. In the following we summarize some 


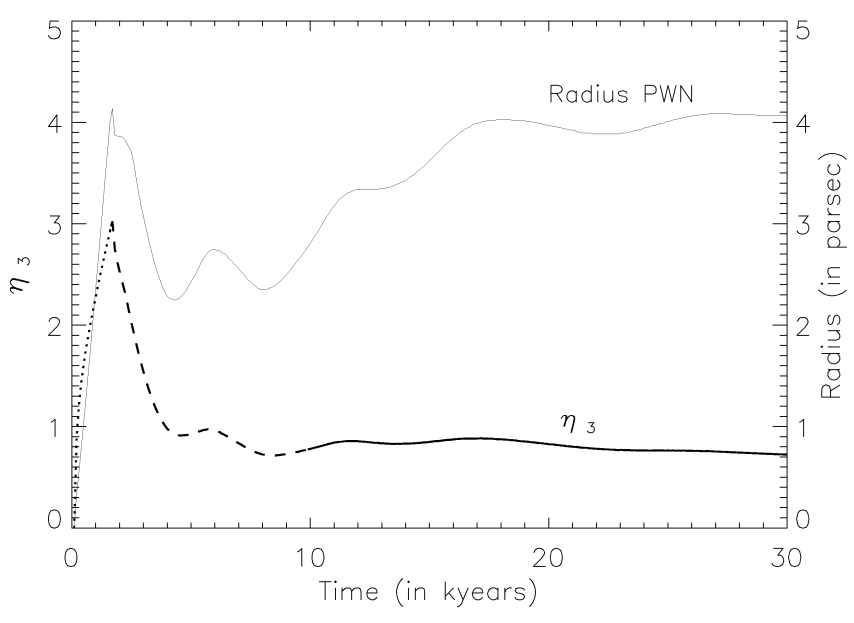

Fig. 1.-Results of numerical simulation showing the time evolution of the PWN. Plotted here are its radius (in units of parsecs; thin continuous line) and the dimensionless parameter $\eta_{3}$ (thick line). The latter is dissected into three parts: a supersonic expansion stage when the PWN is bounded by a shock (dotted section), an unsteady transition when undergoing reverberation with the supernova reverse shock (dashed section), and subsonic expansion (continuous section). The parameters adopted in this simulation are maximum spindown luminosity $L_{0}=5 \times 10^{38} \mathrm{ergs} \mathrm{s}^{-1}$, spin-down time $\tau=600 \mathrm{yr}$, supernova explosion energy of $E_{0}=10^{51} \mathrm{ergs}$, and ISM density of $10^{-24} \mathrm{~g} \mathrm{~cm}^{-3}$. We find $t_{\mathrm{ST}} \sim 10^{3} \mathrm{yr}$ in this case. Note that $\eta_{3}$ is not physical in the early expansion stages but is only formally defined by eq. (5). For details, see van der Swaluw et al. (2001).

relevant results from Chevalier \& Fransson (1992) and van der Swaluw et al. (2001).

\subsection{Analytical Relations}

We focus on the subsonic expansion stage of the PWN, which occurs when the SNR has relaxed to the Sedov solution. We assume that the pulsar wind has deposited most of its energy into the PWN $(t \gg \tau)$. We find that the radius of the PWN $\left(R_{\mathrm{pwn}}\right)$ scales roughly linearly with the radius of the SNR $\left(R_{\mathrm{snr}}\right)$. This scaling arises from the condition of pressure equilibrium between the PWN interior and the interior of the SNR. We approximate the pressure that confines the PWN by the central pressure of the SNR, using the Sedov solution (Sedov 1959),

$$
P_{\mathrm{snr}} \simeq 0.074 E_{0} / R_{\mathrm{snr}}^{3},
$$

where $E_{0}$ is the total mechanical energy of the SNR. Inside the PWN, pressure is quickly equilibrated because of the high sound speed $\sim c / \sqrt{3}$ of the relativistic fluid. The interior pressure is related to the energy content of the PWN, $E_{*}$, by

$$
P_{\mathrm{pwn}} \simeq \frac{3(\gamma-1)}{4 \pi} \frac{E_{*}}{R_{\mathrm{pwn}}^{3}}
$$

where $\gamma$ is the adiabatic index. Let $E_{\mathrm{pwn}}$ be the total amount of energy injected into the PWN by the central pulsar. Part of this energy is used to perform work on the surrounding medium as the PWN expands. Despite some compression of the PWN by the reverse shock around $t=t_{\mathrm{ST}}, E_{*}$ falls below $E_{\mathrm{pwn}}$ (see Fig. 9 of van der Swaluw et al. 2001). We therefore write $E_{*}=\eta_{2} E_{\mathrm{pwn}}$ with $\eta_{2}<1$ decreasing over time. Imposing pres- sure equilibrium, we find

$$
R_{\mathrm{pwn}}=\bar{C}\left(E_{*} / E_{0}\right)^{1 / 3} R_{\mathrm{snr}},
$$

where $\bar{C} \simeq 1.02$ for a relativistic fluid $(\gamma=4 / 3)$ and $\bar{C} \simeq 1.29$ for a nonrelativistic fluid $(\gamma=5 / 3)$.

In the absence of radiative losses, the subsonic expansion of the PWN is adiabatic, $P_{\mathrm{pwn}} V_{\mathrm{pwn}}^{\gamma}=$ constant, where $V_{\mathrm{pwn}} \propto R_{\mathrm{pwn}}^{3}$ is the volume of the PWN. Combining this relation with equation (3), we find that $E_{*}$ falls off with time as $t^{-3 / 10}$ for a relativistic fluid. This yields an expansion law for the radius of the PWN, $R_{\text {pwn }} \propto t^{3 / 10}$, whereas the radius of the SNR scales as in a Sedov solution $\left(R_{\mathrm{snr}} \propto t^{2 / 5}\right)$. Thus, roughly, $R_{\mathrm{pwn}} \propto R_{\mathrm{snr}}$.

We introduce two additional dimensionless parameters, $\eta_{1}$ and $\eta_{3}$, and rewrite equation (4) into a form that can be more easily compared with simulations,

$$
R_{\mathrm{pwn}}(t)=\eta_{3}(t)\left(\eta_{1} E_{\mathrm{sd}} / E_{0}\right)^{1 / 3} R_{\mathrm{snr}}(t)
$$

The parameter $\eta_{1}$ is a constant and relates the total energy input into the PWN, $E_{\mathrm{pwn}}$, to the total spin-down energy of the pulsar $E_{\mathrm{sd}}$ as $E_{\mathrm{pwn}}=\eta_{1} E_{\mathrm{sd}}$. This takes into account possible radiative losses in the PWN and other inefficiencies in the conversion of the spin-down energy into mechanical energy. For instance, a fraction of the neutron star spin-down energy may escape directly from the pulsar as high-energy radiation. We set $\eta_{1}=1$ in this Letter but discuss the case when it is much less than unity. The second parameter is defined as $\eta_{3}(t)=\bar{C} \eta_{2}^{1 / 3} \leq \bar{C}$. The maximum value of $\eta_{3}=\bar{C}$ is used when we determine the initial spin periods of pulsars driving composite remnants.

The derivation leading up to equation (5) is invalid during the earlier supersonic evolution. We retain equation (5) with the sole purpose of defining $\eta_{3}$ during these stages. We proceed to demonstrate the behavior of $\eta_{3}$ throughout the PWN evolution with results from hydrodynamical simulations.

\subsection{Hydrodynamical Simulations}

Van der Swaluw et al. (2001) have presented hydrodynamical simulations of a pulsar wind nebula inside an SNR. In these simulations, the gasdynamical equations are integrated in a spherically symmetric configuration, using the versatile advection code developed by G. Tóth at the Astronomical Institute, Utrecht (Tóth \& Odstrčil 1996). Lacking the possibility of treating a relativistic fluid, the pulsar wind has been implemented as a cold nonrelativistic wind $(\gamma=5 / 3)$ with a terminal velocity equal to the speed of light, $v_{\infty}=\left[2 L(t) / \dot{M}_{\mathrm{pw}}(t)\right]^{1 / 2} \simeq c$, where $\dot{M}_{\mathrm{pw}}(t)$ is the mass ejection rate into the pulsar wind bubble and $L(t)$ is the pulsar spin-down luminosity as given by equation (1).

Figure 1 presents relevant results from one of these simulations. The numerical parameters adopted in this simulation are listed in the figure legend. Taking different parameters will not qualitatively change the overall behavior of the system, but it will affect the early (supersonic) evolution and the moment when subsonic expansion commences $\left(t_{\mathrm{ST}}\right)$. For example, $t_{\mathrm{ST}}$ is larger if the supernova explosion is more energetic, if the supernova ejecta mass is larger, or if the interstellar medium density is smaller $\left(t_{\mathrm{ST}} \propto E_{0}^{-1 / 2} M_{\mathrm{ej}}^{5 / 6} \rho_{\mathrm{ism}}^{-1 / 3}\right.$; e.g., McKee \& Truelove 1995). Since this is a nonrelativistic simulation $(\gamma=$ $5 / 3$ ), we expect $\eta_{3} \leq \bar{C}=1.29$ in the subsonic expansion stage. This is indeed observed. Therefore, in the following investigation we assume a maximum value for $\eta_{3}$ of 1.02 , as is ap- 
TABLE 1

List of Composite REMNANTS

\begin{tabular}{|c|c|c|c|c|c|c|c|}
\hline \multirow[b]{2}{*}{$\begin{array}{c}\text { SUPERNOVA } \\
\text { DESIGNATION }\end{array}$} & \multicolumn{3}{|c|}{ PleRION } & \multicolumn{3}{|c|}{ PULSAR } & \multirow[b]{2}{*}{$\begin{array}{l}\text { AGE } \\
(\mathrm{kyr})\end{array}$} \\
\hline & $R_{\mathrm{pwn}} / R_{\mathrm{snr}}$ & Reference & $\begin{array}{c}P_{0} \\
(\mathrm{~ms})\end{array}$ & $\begin{array}{c}P_{t} \\
(\mathrm{~ms})\end{array}$ & $\begin{array}{c}P_{0}^{\prime} \\
(\mathrm{ms})\end{array}$ & Reference & \\
\hline $\mathrm{G} 16.7+0.1$ & 0.25 & Helfand et al. 1989 & 43 & & $\ldots$ & $\ldots$ & \\
\hline G29.7-0.3 $\ldots \ldots \ldots \ldots$ & 0.16 & Becker \& Helfand 1984 & 82 & 325 (PSR 1846-0258) & $\cdots$ & $\ldots$ & 0.7 \\
\hline G34.7-0.4 $\ldots \ldots \ldots \ldots$ & 0.07 & Frail et al. 1996 & 197 & 267 (PSR 1853+01) & $\ldots$ & $\ldots$ & $\ldots$ \\
\hline $\mathrm{G} 293.8+0.6 \ldots \ldots \ldots \ldots$ & 0.25 & Whiteoak \& Green 1996 & 43 & $\ldots$ & $\ldots$ & $\ldots$ & $\ldots$ \\
\hline G327.1-1.1 $\ldots \ldots \ldots$ & 0.25 & Sun, Wang, \& Chen 1999 & 43 & $\ldots$ & $\ldots$ & $\ldots$ & $\ldots$ \\
\hline $\mathrm{G} 351.2+0.1 \ldots \ldots \ldots \ldots$ & 0.05 & Becker \& Helfand 1988 & 484 & & . & $\ldots$ & \\
\hline G263.9-3.3 $\ldots \ldots \ldots \ldots$ & 0.24 & Milne 1980 & 41 & 89 (Vela) & 52 & Lyne et al. 1996 & 11.3 \\
\hline G11.2-0.3* & 0.15 & Vasisht et al. 1996 & 53 & 65 & 63 & Torii et al. 1999 & 1.6 \\
\hline $\mathrm{G} 320.4-1.2 *$ & 0.17 & Seward et al. 1983 & 69 & 150 (PSR B1509-58) & 63 & Kaspi et al. 1994 & 1.5 \\
\hline SNR $0540-69.3 \ldots \ldots$ & 0.08 & Manchester et al. 1993 & 49 & 50.3 (PSR 0540-69) & 39 & Deeter, Nagase, \& Boynton 1999 & 0.8 \\
\hline
\end{tabular}

Note. $-P_{0}$ is the initial spin period from the radius method, $P_{t}$ is the current spin period of systems with identified pulsars, and $P_{0}^{\prime}$ is the calculated initial spin period by using the braking index.

propriate for the subsonic expansion stage of a PWN in an SNR with $\gamma=4 / 3$.

\section{INFERRING INITIAL SPIN RATES}

Writing the spin-down energy as $E_{\mathrm{sd}}=\left(\Omega_{0}^{2}-\Omega_{t}^{2}\right) I / 2$, we obtain the following expression for the pulsar's initial spin period:

$$
P_{0}=2 \pi\left[\frac{2 E_{0}}{\eta_{1} I}\left(\frac{R_{\mathrm{pwn}}}{\eta_{3} R_{\mathrm{snr}}}\right)^{3}+\left(\frac{2 \pi}{P_{t}}\right)^{2}\right]^{-1 / 2}
$$

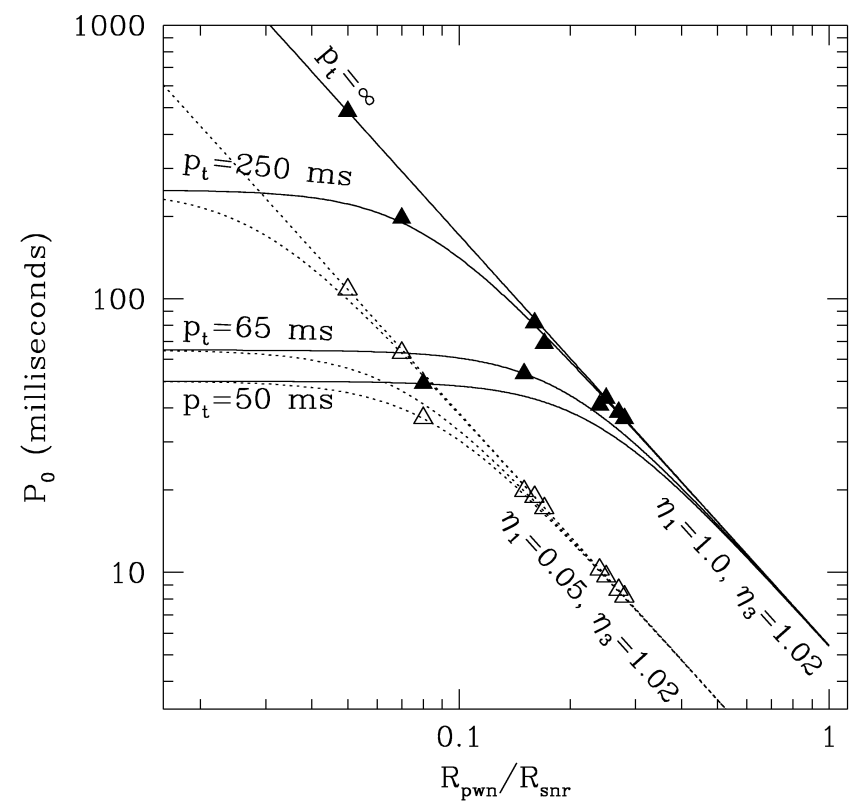

FIG. 2.-Initial spin periods inferred from the ratio $R_{\mathrm{pwn}} / R_{\mathrm{snr}}$ using eq. (6). When assuming no radiative loss $\left(\eta_{1}=1\right)$, we obtain solutions as depicted by the family of solid lines, with different lines applicable for different current spin periods, $P_{t}$. The family of dashed lines are for $\eta_{1}=0.05$. Filled and open triangles represent systems listed in Table 1 for $\eta_{1}=1$ and $\eta_{1}=0.05$, respectively. When $P_{0} \leq 4 \mathrm{~ms}$, the initial rotational energy of the pulsar exceeds the total mechanical energy in the SNR itself. Eq. (6) fails, and the SNR may be blown away by the PWN. None of the systems we examined lie close to this limit. where the spin periods are $P_{0} \equiv 2 \pi / \Omega_{0}$ and $P_{t} \equiv 2 \pi / \Omega_{t}$ and $I$ is the moment of inertia of the neutron star. The radius of a PWN relative to its associated SNR shell can be used to infer the initial spin period of the pulsar, assuming $\eta_{1}, \eta_{3}, E_{0}$, and $\Omega_{t}$ are known or can be estimated.

The radii of PWNs should be taken from radio measurements. Synchrotron-radiating electrons in PWNs have a typical lifetime (Rybicki \& Lightman 1979)

$$
\tau_{1 / 2}=\frac{6 \pi m_{e} c}{\sigma_{\mathrm{T}} B^{2} \Gamma} \sim 10^{5} B_{\mathrm{mG}}^{-3 / 2} \nu_{\mathrm{MHz}}^{-1 / 2} \mathrm{yr},
$$

where $\sigma_{\mathrm{T}}$ is the Thomson cross section, $\Gamma$ is the electron Lorentz factor, $\nu$ is the typical synchrotron frequency scaled at the radio wavelength, and $B$ is the magnetic field strength scaled by milligauss, a value appropriate for plerions. Such a long lifetime implies that radio observations "see" electrons that were accelerated during the early energetic stage when the pulsar deposits most of its rotational energy into the pulsar wind. They therefore delineate the true spatial extent of the plerion. In contrast, X-ray electrons lose their energy quickly. The X-ray plerion is produced by electrons recently accelerated near the pulsar, so one expects the plerion to be smaller in X-rays.

We have collected from the literature 13 composite SNRs. We list the relevant properties of these systems in Table 1. The relative sizes of the plerions are taken from radio observations, with the exception of G11.2-0.3 and G320.4-1.2 (marked by asterisks), where only X-ray plerions have been observed. We apply equation (6) to determine the initial spin periods for these systems, adopting $\eta_{1}=1.0, \eta_{3}=1.02, E_{0}=1.0 \times 10^{51} \mathrm{ergs}$, $I=1.4 \times 10^{45} \mathrm{~g} \mathrm{~cm}^{2}$ (the value for $\mathrm{Crab}$ ), and $P_{t}=$ $2 \pi / \Omega_{t}=\infty$ in cases where this value is unknown. The same results appear in Figure 2 as filled triangles. By taking the maximum value for $\eta_{3}$, we probably underestimate the initial spin rate, but likely by no more than a factor of 2 judging from Figure 1. In the same table we also list the initial spin periods $\left(P_{0}^{\prime}\right)$ derived for four pulsars using braking index measurements.

\section{DISCUSSION}

We have demonstrated a new method for inferring initial spin rates of pulsars residing in composite SNRs. This method uses the ratio of the plerion radius and the radius of the su- 
pernova shell, so we dub it the "radius method." Given that typical plerions and SNRs are expected to live for $10^{3}-10^{4} \mathrm{yr}$, we expect that future observations will increase the population to which the radius method can be applied.

This method does not require a knowledge of parameters like the density and magnetic field strength of the ISM or the distance and age of the SNR. However, for the method to apply, the observed PWN has to be in the subsonic stage of its evolution. If this is not the case, the method systematically overestimates the initial spin rate (see below). Uncertainties in $E_{0}, \eta_{3}$, and $\Omega_{t}$ introduce errors of order unity, as will a deviation from pure spherical symmetry. A more important uncertainty of this new method concerns the influence of synchrotron radiative losses in the plerion, as parameterized by $\eta_{1}$. We tend to underestimate the initial spin rate by ignoring these losses (setting $\eta_{1}=1$ ). We will illustrate the effect of these uncertainties by considering the four systems in Table 1, which have known central pulsars, and independent estimates for their initial periods based on measurements of the pulsar braking index. ${ }^{4}$

Among these systems, Vela is the oldest and should have entered the subsonic expansion stage. Its associated pulsar being displaced from the center of the plerion also testifies in favor of an old age. Adopting $\eta_{3}=1.02$ and other parameters as stated, the radius method yields an initial spin-down energy for Vela that is a factor of 2 above that inferred by Lyne et al. (1996) from pulsar braking index measurements. This is surprising because we have ignored radiative loss. We suggest three possibilities that will reconcile the two methods: (1) the supernova explosion is one-half as energetic as assumed here, (2) the pulsar is $15 \mathrm{kyr}$ old, and (3) the braking index is as high as 2.4 (Lyne et al. 1996 reported 1.4). The first option will raise $P_{0}$ to $52 \mathrm{~ms}$, while the latter two will reduce $P_{0}^{\prime}$ to $41 \mathrm{~ms}$.

The remaining two systems, G11.2-0.3 and G320.4-1.2, are young $(\sim 1.5 \mathrm{kyr})$. Their plerions could still be in supersonic expansion during which the value of $\eta_{3}$ (defined formally through eq. [5] in the supersonic stage) can be higher than 1.02, the theoretical maximum during the subsonic expansion stage (Fig. 1 and $\S 2$ of van der Swaluw et al. 2001). For example, taking $\eta_{3}=2$, we find $P_{0}=63 \mathrm{~ms}$ for G11.2-0.3. A similar correction may have to be applied to G29.7-0.3 (Kes 75).

\footnotetext{
${ }^{4}$ G11.2-0.3 does not have a braking index measurement. However, its inferred initial spin depends little on this index because of its small age.
}

In contrast to the systems discussed above, SNR 0540-69.3 is the only case in which the spin-down energy is significantly underestimated for our choice of parameters. The plerion in SNR 0540-69.3 is too small if the pulsar was indeed born with an initial spin of 39 ms. Manchester, Staveley-Smith, \& Kesteven (1993) have argued that the radiative loss in this system has reduced the total energy stored in the plerionic magnetic field and relativistic electrons to a mere $4 \%$ of the integrated spindown luminosity of the pulsar. Taking $\eta_{1}=0.04$, we recover a $39 \mathrm{~ms}$ initial period, with a value of $\eta_{3}=1.3$. This value of $\eta_{3}$ is reasonable because SNR 0540-69.3 is only $760 \mathrm{yr}$ old. None of the other three systems discussed here require strong synchrotron losses in the plerion, which makes SNR 0540-69.3 unique. Among the four pulsars, PSR 0540-69 is not outstanding either in its surface magnetic field strength or in its spindown rate. It is likely born the fastest, though not by a large margin.

Along the same lines, Atoyan (1999) has argued that, in Crab, strong synchrotron losses in its past can account for the observed flat spectra of the radio electrons in the plerion. If this applied to all PWNs, it would significantly change our inferred initial spin rates. This is illustrated in Figure 2 for a strong synchrotron loss of $\eta_{1}=0.05$.

Although the effect of radiative losses on the reliability of our results remains unclear, we briefly explore the implications of these results. Our sample (Table 1) is likely biased toward fast spinners, which produce plerions that are easier to detect. Even so, all pulsars in our sample seem to be born spinning much below the breakup rate. Moreover, their initial spin periods do not cluster around $\sim 5-10 \mathrm{~ms}$, a signature of rapid spin-down by the gravitationally radiating Rossby waves, which occur immediately after the supernova collapse (Andersson et al. 2000; Wu, Matzner, \& Arras 2001). The long initial periods we find imply an effective angular momentum coupling between the cores and envelopes of neutron star progenitors until late into their evolution. The scattering in the initial periods could either reflect a different decoupling time in different stars or a stochastic process that gives rise to the final angular momentum in the neutron star, such as an offcentered kick during the collapse (Spruit \& Phinney 1998).

We acknowledge discussions with Bram Achterberg, Armen Atoyan, and an anonymous referee.

\section{REFERENCES}

Andersson, N., Jones, D. I., Kokkotas, K. D., \& Stergioulas, N. 2000, ApJ, 534, L75

Atoyan, A. M. 1999, A\&A, 346, L49

Becker, R. H., \& Helfand, D. J. 1984, ApJ, 283, 154 . 1988, AJ, 95, 883

Caraveo, P. A., \& Mignani, R. P. 1999, A\&A, 344, 367

Chevalier, R. A., \& Fransson, C. 1992, ApJ, 395, 540

Deeter, J. E., Nagase, F., \& Boynton, P. E. 1999, ApJ, 512, 300

Emmering, R. T., \& Chevalier, R. A. 1989, ApJ, 345, 931

Frail, D. A., Giacani, E. B., Goss, W. M., \& Dubner, G. 1996, ApJ, 464, L165

Haensel, P., Salgado, M., \& Bonazzola, S. 1995, A\&A, 296, 745

Harrison, E. R., \& Tademaru, E. 1975, ApJ, 201, 447

Heger, A., Langer, N., \& Woosley, S. E. 2000, ApJ, 528, 368

Helfand, D. J., \& Becker, R. H. 1987, ApJ, 314, 203

Helfand, D. J., Velusamy, T., Becker, R. H., \& Lockman, F. J. 1989, ApJ, 341, 151

Kaspi, V. M., Manchester, R. N., Siegman, B., Johnston, S., \& Lyne, A. G. 1994, ApJ, 422, L83

Kassim, N. E., Hertz, P., \& Weiler, K. W. 1993, ApJ, 419, 733

Lai, D., Chernoff, D. F., \& Cordes, J. M. 2001, ApJ, 549, 1111

Lai, D., \& Goldreich, P. 2000, ApJ, 535, 402

Lindblom, L., Owen, B. J., \& Morsink, S. M. 1998, Phys. Rev. Lett., 80, 4843
Lorimer, D. R., Bailes, M., Dewey, R. J., \& Harrison, P. A. 1993, MNRAS, 263, 403

Lyne, A. G., Pritchard, R. S., \& Graham-Smith, F. 1993, MNRAS, 265, 1003

Lyne, A. G., Pritchard, R. S., Graham-Smith, F., \& Camilo, F. 1996, Nature, 381,497

Manchester, R. N., Staveley-Smith, L., \& Kesteven, M. J. 1993, ApJ, 411, 756

Marshall, F. E., Gotthelf, E. V., Zhang, W., Middleditch, J., \& Wang, Q. D. 1998, ApJ, 499, L179

McKee, C. F., \& Truelove, J. K. 1995, Phys. Rep., 256, 157

Milne, D. K. 1980, A\&A, 81, 293

Narayan, R. 1987, ApJ, 319, 162

Pavlov, G. G., Sanwal, D., Garmire, G. P., Zavlin, V. E., Burwitz, V., \& Dodson, R. G. 2000, AAS Meeting, 196, 3704

Phinney, E. S., \& Blandford, R. D. 1981, MNRAS, 194, 137

Reynolds, S. P., \& Chevalier, R. A. 1984, ApJ, 278, 630

Rybicki, G. B., \& Lightman, A. P. 1979, Radiative Processes in Astrophysics (New York: Wiley)

Sedov, L. I. 1959, Similarity and Dimensional Methods in Mechanics (New York: Academic)

Seward, F. D., Harnden, F. R., Murdin, P., \& Clark, D. H. 1983, ApJ, 267, 69

Spruit, H. C., \& Phinney, E. S. 1998, Nature, 393, 139 
Srinivasan, G., Dwarakanath, K. S., \& Bhattacharya, D. 1984, J. Astrophys. Astron., 5, 403

Sun, M., Wang, Z., \& Chen, Y. 1999, ApJ, 511, 274

Torii, K., Tsunemi, H., Dotani, T., Mitsuda, K., Kawai, N., Kinugasa, K., Saito, Y., \& Shibata, S. 1999, ApJ, 523, L69

Tóth, G., \& Odstrčil, D. 1996, J. Comput. Phys., 128, 82

van der Swaluw, E., Achterberg, A., Gallant, Y. A., \& Tóth, G. 2001, A\&A, submitted (astro-ph/0012440)
Vasisht, G., Aoki, T., Dotani, T., Kulkarni, S. R., \& Nagase, F. 1996, ApJ, 456, L59

Vivekanand, M., \& Narayan, R. 1981, J. Astrophys. Astron., 2, 315

Weiler, K. W., \& Panagia, N. 1978, A\&A, 70, 419

Whiteoak, J. B. Z. 1992, MNRAS, 256, 121

Whiteoak, J. B. Z., \& Green, A. J. 1996, A\&AS, 118, 329

Wu, Y., Matzner, C. D., \& Arras, P. 2001, ApJ, 549, 1011 